\title{
Antimicrobial Activity and Proposed Action Mechanism of Linalool Against Pseudomonas fluorescens
}

\author{
Fengyu Guo, Qianping Chen, Qiong Liang, Ming Zhang, Wenxue Chen, Haiming Chen, \\ Yonghuan Yun, Qiuping Zhong* and Weijun Chen*
}

College of Food Science and Technology, Hainan University, Haikou, China

\section{OPEN ACCESS}

Edited by:

Octavio Luiz Franco,

Catholic University of Brasilia (UCB),

Brazil

Reviewed by:

Christopher Auger,

Sunnybrook Health Science Centre,

Canada

Mariana Rocha Maximiano, Universidade Católica Dom Bosco,

Brazil

*Correspondence:

Qiuping Zhong

hainufood88@163.com

Weijun Chen

chenwj@nwu.edu.cn

Specialty section:

This article was submitted to Antimicrobials, Resistance

and Chemotherapy,

a section of the journa

Frontiers in Microbiology

Received: 15 May 2020 Accepted: 06 January 2021

Published: 28 January 2021

Citation:

Guo F, Chen Q, Liang Q, Zhang $M$, Chen W, Chen $H$, Yun Y,

Zhong $Q$ and Chen W (2021) Antimicrobial Activity and Proposed Action Mechanism of Linalool Against

Pseudomonas fluorescens.

Front. Microbiol. 12:562094.

doi: 10.3389/fmicb.2021.562094
In this study, linalool, one of the principal components of essential oils, was used as an antibacterial agent to investigate the antibacterial activity and mechanism of linalool against Pseudomonas fluorescens. The reduction in membrane potential (MP), leakage of alkaline phosphatase (AKP) and the release of macromolecules, including DNA, RNA and protein confirmed that damage to cell wall membrane structure and leakage of cytoplasmic contents were due to the linalool treatment. Furthermore, the decrease of enzyme activity, including the succinate dehydrogenase (SDH), malate dehydrogenase $(\mathrm{MDH})$, pyruvate kinase $(\mathrm{PK})$, and ATPase indicated that linalool could lead to metabolic dysfunction and inhibit energy synthesis. In addition, the activity of respiratory chain dehydrogenase and metabolic activity of respiration indicated that linalool inhibits cellular respiration. These results revealed that linalool had strong antibacterial activity against $P$. fluorescens via membrane damage, bacterial metabolic and oxidative respiratory perturbations, interfering in cellular functions and even causing cell death. It was suggested that linalool may be a new potential source as food antiseptics in food systems.

Keywords: linalool, antibacterial mechanism, Pseudomonas fluorescens, membrane damage, metabolic respiration, TCA cycle

\section{INTRODUCTION}

In recent years, aromatic plant extracts have been widely used in the field of food preservation (Brenes and Roura, 2010; Prakash et al., 2015). Essential oils (EOs) are naturally occurring antimicrobial agents found in many plants and are the main reason for the antimicrobial properties of aromatic plant extracts (Brenes and Roura, 2010; Callaway et al., 2011). At present, many of the most widely used food preservatives are synthetic products. However, synthetic products have many disadvantages, such as potential carcinogenicity, teratogenicity, environmental pollution, and acute toxicity (Faleiro, 2011). Therefore, people are eager to find natural food additives with which to replace the synthetic chemical additives (Fratianni et al., 2010). EOs are an ideal choice because of their significant antibacterial properties and safety (Calo et al., 2015).

Linalool $\left(\mathrm{C}_{10} \mathrm{H}_{18} \mathrm{O}\right)$, also known as 3,7-dimethyl-1,6-octadien-3-ol, is a monoterpene alcohol, which is found in the EOs extracted from more than 200 plants worldwide, such as Thymus vulgaris (thyme) EOs and Juniperus communis (juniper) EOs (Aprotosoaie et al., 2014; Pereira et al., 2018). This monoterpene alcohol poses antioxidant, anti-inflammatory, and anticancer activities (Herman et al., 2016) and exhibits antibacterial activity against Staphylococcus aureus 
NCTC 10788, Pseudomonas aeruginosa NCTC 12924, and Escherichia coli NCTC 12923 (Herman et al., 2016). Consequently, it is widely used in pharmaceuticals, cosmetics and food additives (Calo et al., 2015). Annually, approximately 1,000 metric tons of linalool are consumed globally each year (Aprotosoaie et al., 2014). In this case, it is very promising to develop natural antimicrobials from linalool.

Pseudomonas fluorescens is a gram-negative bacterium, represented by a single-celled rod with a curved or straight long axis, one or more polar flagella, no spore formation, sheath, or stem motility (Kumar et al., 2019). P. fluorescens survives well at ambient temperature, and can easily contaminate refrigerated food which affects the flavor and quality of the food and is a serious threat to the food industry (Kumar et al., 2019). In addition, $P$. fluorescens are associated with some human diseases, such as septicemia, septic shock, and intravascular coagulation (Quintieri et al., 2019; Shu et al., 2019). An investigation in Italy reported that ready-to-eat vegetables were contaminated with P. fluorescens (Caldera and Franzetti, 2013). Another example is pasteurized milk that for a short time can still be contaminated with $P$. fluorescens, which produces luciferin that seriously affects the flavor and quality of the milk (Reichler et al., 2018).

Extensive research has been done on the antibacterial activity of linalool (Jin et al., 2020; Liu et al., 2020), but to the best of the authors' knowledge, the mechanism underlying the antibacterial activity of linalool against $P$. fluorescens remains poorly understood. Therefore, the present study aimed to characterize the antibacterial properties of linalool and to investigate the mechanism of its antibacterial action against $P$. fluorescens. The antibacterial mechanism of linalool was investigated from two aspects: (1) The effects of linalool on the cell morphology, which mainly include the membrane potential, and intracellular macromolecular substances. (2) The effects of linalool on the intracellular metabolic activities of the bacterium which mainly includes measuring the tricarboxylic acid cycle, the glycolysis pathway, and the activities of enzymes related to respiration.

\section{MATERIALS AND METHODS}

\section{Materials and Chemicals}

A strain of P. fluorescens (ATCC13525) was purchased from the Guangdong Microbial Culture Preservation Center (Guangdong, China), and incubated in a nutrient broth $\left(30^{\circ} \mathrm{C}, 150 \mathrm{rpm}\right)$ for $24 \mathrm{~h}$ to obtain the log-phase of the bacteria. Linalool was purchased from the Hainan Camphora Biotech Co., Ltd. (Hainan, China). Rhodamine 123, resazurin, and iodonitrotetrazolium chloride (INT) were purchased from Shanghai Yuanye Bio-Technology Co., Ltd. (Shanghai, China). The bicinchoninic acid (BCA), adenosine triphosphatase (ATPase) A070-2-2, malic acid dehydrogenase (MDH) A0212-1, succinic acid dehydrogenase (SDH) A022-1-1, and pyruvate kinase (PK) A076-1-1 assay kits were purchased from Nanjing JianCheng Bioengineering Institute (Nanjing, China). Unless otherwise specified, all other chemicals were of an analytical grade.

\section{The Antibacterial Activity of Linalool \\ Determination of the Minimal Inhibitory Concentrations (MIC), and Minimum Bactericidal Concentration (MBC) of Linalool}

The MIC and MBC of linalool were determined using the agar dilution method (Shu et al., 2019). Briefly, linalool was mixed with ethanol (10\% final concentration of ethanol), and then $2 \mathrm{~mL}$ of this solution was added to $18 \mathrm{~mL}$ of solid medium to obtain antibacterial solutions with final concentrations of $0.3125,0.625$, $1.25,2.5$, and $5 \mu \mathrm{l} / \mathrm{mL}$, respectively. Next, a prepared plate was coated with $200 \mu \mathrm{L}$ of the activated bacterial suspension, and the plate was incubated at $30^{\circ} \mathrm{C}$ for $24 \mathrm{~h}$. A sample with sterilized water was used as the blank control, and ethanol (1\%) was added to a suspension of the bacterial for the negative control. The experiment was repeat three times for each concentration gradient. The MIC value was the lowest concentration of linalool which visibly inhibited the growth of the bacteria. The MBC value was the lowest concentration of linalool at which no colony was visible on the surface after $48 \mathrm{~h}$ of continuous culture.

\section{The Growth Curve of $P$. fluorescens}

The antibacterial activity of linalool was reflected in the growth curve of P. fluorescens (Zeng et al., 2011; Zhang et al., 2017). Activated $P$. fluorescens was diluted to $1 \times 10^{6-7} \mathrm{CFU} / \mathrm{mL}$, and linalool was diluted with $1 \%$ ethanol and added to the medium to make the final concentration 1/2MIC and 1MIC. One millilitre of the bacterial suspension was mixed with $100 \mathrm{~mL}$ of the prepared medium and all cultures were incubated at $30^{\circ} \mathrm{C}$ for $24 \mathrm{~h}$. Three parallel samples were taken from each group every $2 \mathrm{~h}$ and the OD $600 \mathrm{~nm}$ value was measured with an ultraviolet spectrophotometer. The OD $600 \mathrm{~nm}$ absorbance value was used to measure the concentration of the bacteria in the solution and reflected the growth rate of the bacteria.

\section{Scanning Electron Microscopy (SEM) Observation}

To determine the efficacy of the essential oil and the morphological changes of $P$. fluorescens strains, SEM observation was used on the tested bacteria (Shu et al., 2019). The $P$. fluorescens log-phase was centrifuged and washed twice with $0.1 \mathrm{M}$ phosphate buffer solution (PBS, pH 7.4), then 1/2 MIC and $1 \mathrm{MIC}$ of linalool were added to the resuspensions, respectively. Control group was added with the same amount of sterile water and ethanol (final concentration 1\%). Next the suspensions were incubated at $30^{\circ} \mathrm{C}$ for 4 and $8 \mathrm{~h}$, respectively, and then the suspensions were centrifuged. After this, the cells were dehydrated using sequential exposure per ethanol concentrations ranging from 30 to $100 \%$. The dehydrated samples were prefreezed at $-20^{\circ} \mathrm{C}$ for $2 \mathrm{~h}$ and freeze-dried for $12 \mathrm{~h}$. The samples were then sputter-coated with gold under vacuum and examined by SEM (S-4800, Hitachi, Tokyo, Japan).

\section{The Antibacterial Mechanism of Linalool Against $P$. fluorescens}

A log-phase bacterial suspension was prepared and treated with various concentrations of linalool (sterile water, 1\% ethanol, 
$1 / 2 \mathrm{MIC}$ and $1 \mathrm{MIC})$. All cultures were incubated at $30^{\circ} \mathrm{C}$ and $150 \mathrm{rpm}$. For the subsequent experiments three parallel samples were collected at different time intervals.

\section{Determination of the Membrane Potential (MP)}

The influence of linalool on the membrane potential was evaluated using the method described by Zhang et al. (2016, 2017) and Fang et al. (2019). The prepared bacterial suspension was centrifuged $(6,000 \mathrm{rpm}, 10 \mathrm{~min})$ every $30 \mathrm{~min}$, washed twice with PBS and resuspended. After incubation under dark conditions for $30 \mathrm{~min}$, after which rhodamine 123 with a final concentration of $2 \mu \mathrm{g} / \mathrm{mL}$ was thoroughly mixed into the bacterial suspension. The bacteria suspension was then washed and resuspended with PBS. Then, the fluorescence value was immediately measured at the excitation and emission wavelengths of 480 and $530 \mathrm{~nm}$, respectively.

\section{Loss of $260 \mathrm{~nm}$-Absorbing Materials}

After $0,30,60,90$, and $120 \mathrm{~min}$ of treatment, the bacterial suspension was centrifuged at 4,000 rpm, and the absorbance of the obtained supernatant was determined at $260 \mathrm{~nm}$ using an ultraviolet spectrophotometer (Yang et al., 2015; Diao et al., 2018).

\section{Leakage of Protein From P. fluorescens}

The leakage of protein from the bacterial cells was tested using the Bicinchoninic acid (BCA) Protein Assay Kit (Jiancheng Bioengineering Institute, Jiangsu, China). After incubation for $0,0.5,1,1.5$, and $2 \mathrm{~h}$, the prepared bacterial were centrifuged $(6,000 \mathrm{rpm}, 10 \mathrm{~min})$ and washed three times with PBS. Subsequently, prepared bacteria cells were further resuspended in PBS for ultrasonic processing (power $300 \mathrm{~W}$, interval $1.1 \mathrm{~s}$, $10 \mathrm{~min}$ ) in an ice bath. Finally, the prepared homogenate was used to determine the concentration of protein leaked from the calls using the BCA Protein Assay Kit (Cui et al., 2018; Lin et al., 2018; Hu et al., 2019).

\section{Effect of Linalool on the Respiratory Metabolism of the Cells \\ Metabolic Activity}

The metabolic activity of $P$. fluorescens with different treatments was measured using resazurin (Zhu et al., 2019). Every $30 \mathrm{~min}$, $100 \mu \mathrm{g} / \mathrm{mL}$ of resazurin solution was added to the log-phase of the bacterial suspensions treated with different concentrations of linalool (sterile water, $1 \%$ ethanol, $1 / 2 \mathrm{MIC}$, and $1 \mathrm{MIC}$ ). These solutions were placed in a shaker for $2 \mathrm{~h}$ in the dark. Then, the samples were centrifuged at $10,000 \mathrm{rpm}$ for $4 \mathrm{~min}$, and the supernatant was collected. Finally, the mean fluorescence intensity (MFI) was determined using a fluorescent microplate reader at excitation and emission wavelengths of 560 and $590 \mathrm{~nm}$, respectively.

\section{The Activity of Respiratory Chain Dehydrogenase}

Respiratory chain dehydrogenase converts the colorless iodonitrotetrazolium chloride (INT) to water-insoluble, dark red iodonitrotetrazolium formazan (INF). Subsequently, the activity of the respiratory chain was evaluated using a reducing spectrophotometric value of INF (Gomaa, 2017; Kang et al., 2018). Bacterial cultures at the logarithmic growth stage were centrifuged at 4,000 r/min for $10 \mathrm{~min}$, washed and suspended with sterile normal saline. The bacterial suspension with different treatments was centrifugated $(4,000 \mathrm{rpm}, 10 \mathrm{~min})$, washed three times and then resuspended with sterile normal saline. Subsequently, the $0.01 \mathrm{~mol} / \mathrm{L}$ INT was prepared with methanol and water (1:1). 1,350 $\mu \mathrm{L}$ of the bacterial suspension was added to the $150 \mu \mathrm{L}$ INT solutions to produce final concentrations of INT in solution of $1 \mathrm{mmol} / \mathrm{L}$. The solution was placed at $30^{\circ} \mathrm{C}$ for $30 \mathrm{~min}$ and measured at $630 \mathrm{~nm}$ using an ultraviolet spectrophotometer.

\section{The Activity of Adenosine Triphosphatase (ATPase), Malic Acid Dehydrogenase (MDH), Succinic Acid Dehydrogenase (SDH), and Pyruvate Kinase (PK)}

Different concentrations of linalool (sterile water, $1 \%$ ethanol, $1 / 2 \mathrm{MIC}$, and $1 \mathrm{MIC}$ ) were added to the bacteria cell solutions (approximately $10^{7} \mathrm{CFU} / \mathrm{mL}$ ). All samples were incubated at $30^{\circ} \mathrm{C}$ and $150 \mathrm{rpm}$, then centrifuged and washed three times with normal saline. Subsequently, the prepared bacteria cells were resuspended in normal saline for the ultrasonic processing (power $300 \mathrm{~W}$, interval $1.1 \mathrm{~s}, 10 \mathrm{~min}$ ) in an ice bath. The ATPase, SDH, MDH, and PK activities of P. fluorescens exposed to different concentrations of linalool (sterile water, $1 \%$ ethanol, 1/2 MIC, and 1 MIC) were determined using detection kits (Jiancheng Bioengineering Institute, Jiangsu, China) that were used in accordance with the manufacturer's instructions.

\section{Statistical Analysis}

All experiments were performed in triplicate. Data were expressed as the mean $\pm S D(n=3)$. One-way ANOVA and Duncan's multiple range tests were used to express the significance of differences $(P<0.05)$ between the means.

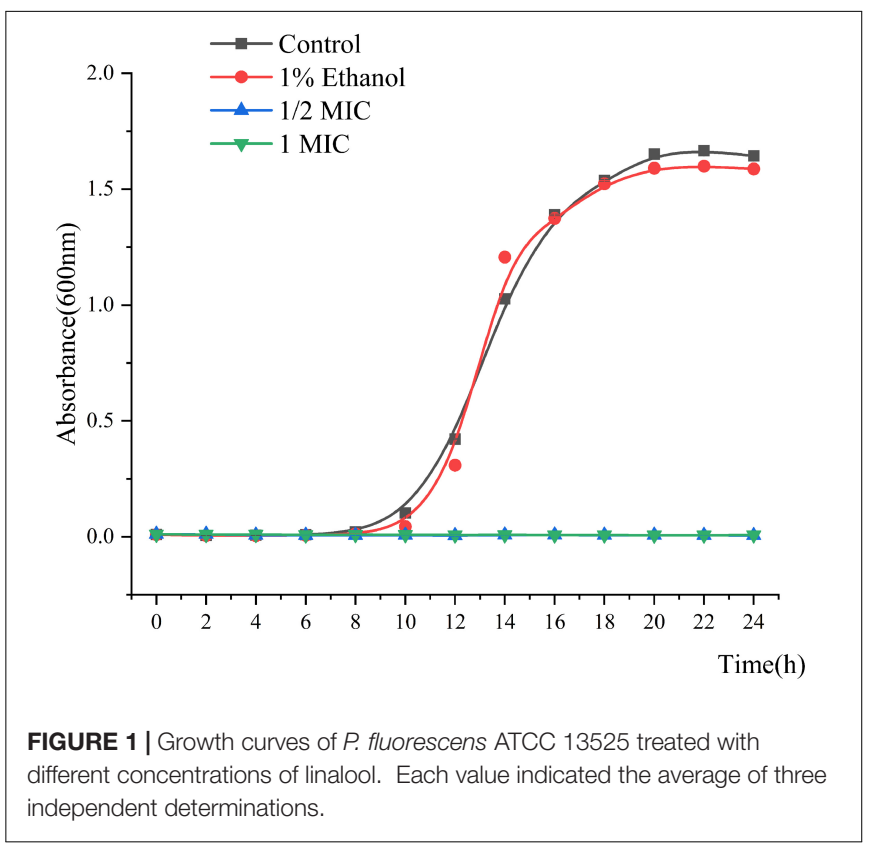




\section{RESULTS}

\section{The Antibacterial Activity of Linalool}

The MIC and MBC of Linalool against $P$. fluorescens was 1.25 and $2.5 \mu \mathrm{L} / \mathrm{mL}$, respectively. As shown in Figure 1, the growth curves of $P$. fluorescens generally followed the model S-shaped growth curve, and reached the logarithmic phase after $6 \mathrm{~h}$ and the stable stage after $18 \mathrm{~h}$. However, the growth of $P$. fluorescens treated with linalool was completely inhibited, indicating that linalool had a good inhibitory effect on P. fluorescens.

\section{The Mechanism by Which Linalool Inhibits $P$. fluorescens \\ Scanning Electron Microscope (SEM)}

The morphological and physical changes of $P$. fluorescens were treated with 1/2MIC and 1 MIC for 2 and 4 h. Figure 2 shows the SEM images of the bacteria in the treated group and the
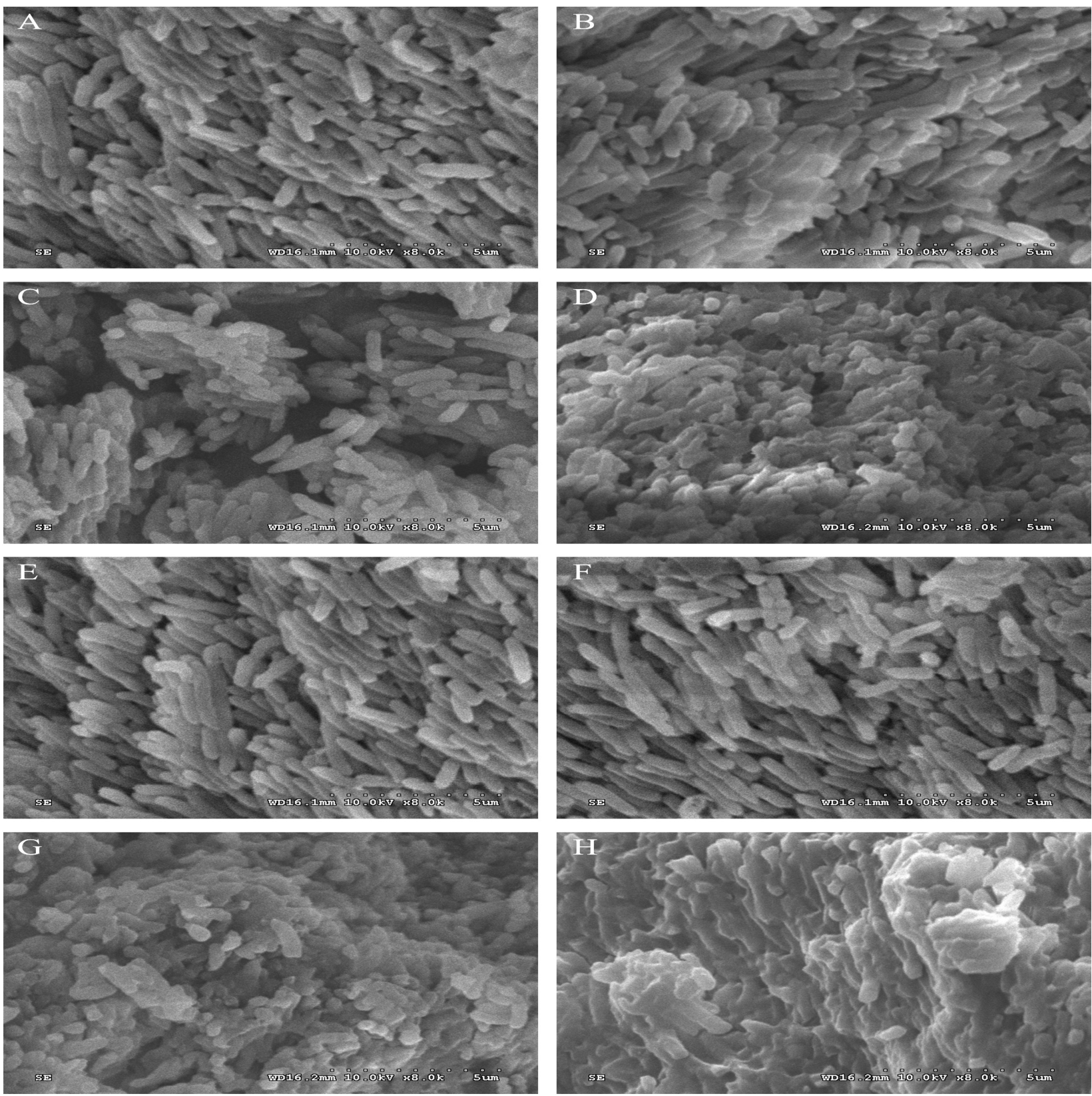

FIGURE 2 | Scanning electron microphotographs of $P$. fluorescens. P. fluorescens untreated for $2 \mathrm{~h}$ (A), untreated for $4 \mathrm{~h}$ (E), treated with $1 \%$ ethanol for $2 \mathrm{~h}$ (B), treated with $1 \%$ ethanol for $4 \mathrm{~h}(\mathbf{F})$, treated with linalool at $1 / 2 \mathrm{MIC}$ for $2 \mathrm{~h}(\mathbf{C})$, treated with linalool at $1 / 2 \mathrm{MIC}$ for $4 \mathrm{~h}(\mathbf{G})$, treated with linalool at $1 \mathrm{MIC}$ for $2 \mathrm{~h}$ (D), treated with linalool at $1 \mathrm{MIC}$ for $4 \mathrm{~h} \mathbf{( H )}$ 
bacteria in the untreated group. These images directly illustrate the damage of linalool to the bacteria. Compared with the untreated control group and 1\% ethanol group, the number of abnormal strains in the processing group increased significantly. Unprocessed cells are bar, rule and complete (Figures 2A,B), while some bacteria that have been processed by linalool have been deformed, wrinkled, glued together, and part of the cell is broken (Figure 2). In addition, 2 and $1 \mathrm{~h}$ of images showed that the damage was obviously increased in the higher concentration of linalool and processing time.

\section{Effect of Linalool on the Membrane Potential (MP)}

The MP was selected as an indicator to evaluate the integrity of the membranes of the cells because it plays an important role in bacterial physiology. The results of the bacterial MP are shown in Figure 3. Compared with the control, P. fluorescens treated with linalool in the $1 / 2$ MIC and 1 MIC groups showed significant cell membrane depolarization because their fluorescent intensity decreased by 63.33 and $68.64 \%$, respectively. The fluorescence intensity of $P$. fluorescens was significantly inversely correlated with the concentration of linalool $(P<0.05)$. This indicated that linalool caused significant depolarization of the cytoplasmic membrane of $P$. fluorescens.

\section{Effect of Linalool on the Nucleic Acids}

The results of $P$. fluorescens treated with linalool for $4 \mathrm{~h}$ (Figure 4A) indicated that the release of the cell constituents were significantly positively correlated with an increase in the concentration of linalool. Compared with the control group, after incubation with $1 / 2 \mathrm{MIC}$ and $1 \mathrm{MIC}$ linalool for $4 \mathrm{~h}$, the levels of nucleic acids (OD $260 \mathrm{~nm}$ ) increased by 2.89 times and 3.46 times $(P<0.05)$, respectively. In addition, the leakage of the intracellular nucleic acids increased significantly with an increase in the concentration of linalool $(P<0.05)$.

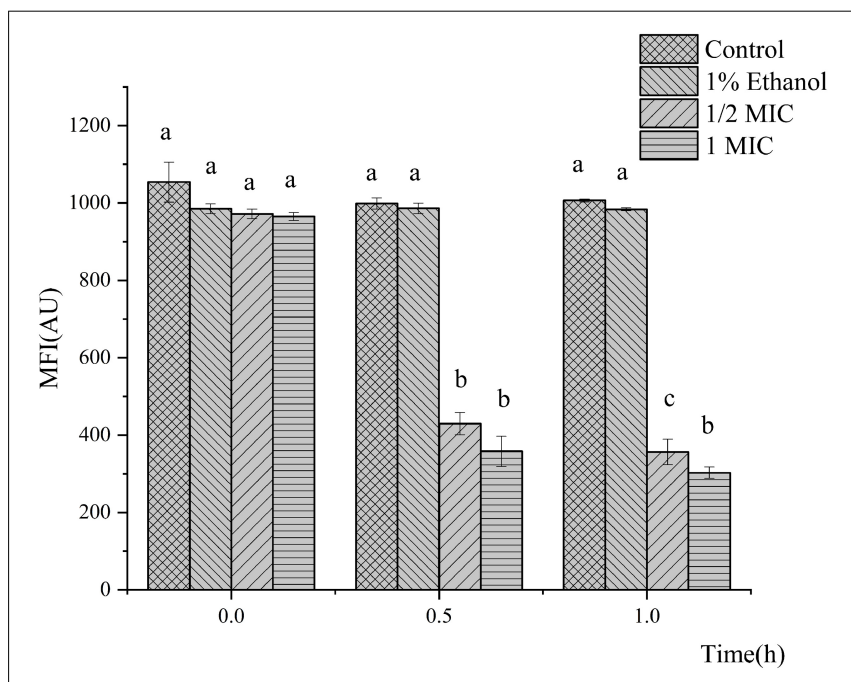

FIGURE 3 | Effect of treatment with linalool on the membrane potential (MP) of $P$. fluorescens. All measurements were expressed as the mean $\pm S E(n=3)$. Means with different letters were significantly different in $\mathrm{MP}(P<0.05)$.

\section{Effect of Linalool on Protein}

Changes in the intracellular protein content can be observed in Figure 4B. Proteins, as important biological macromolecules in cells, usually do not leak out of the cells. Consequently, any change in the intracellular protein content of cells can be used as an indicator of the structural integrity of the cells. In Figure $4 \mathbf{B}$, the protein concentration of 1MIC linalool treated bacteria was $69.42 \mu \mathrm{g} / \mathrm{mL}$ at $2 \mathrm{~h}$. Compared with a protein content of 387.39 $\mu \mathrm{g} / \mathrm{mL}$ for the control group, the protein content after treatment with linalool had decreased by $81.66 \%$. Moreover, this effect of treating linalool $P$. fluorescens with linalool was more significant positively correlated with the concentration of linalool $(P<0.05)$.

\section{Effect of Linalool on Metabolic Activity}

The effect of linalool on the metabolic activity of $P$. fluorescens is shown in Figure 4C. The metabolic activity of the treatment group decreased with an increase in the treatment time, and decreased by approximately 11.18 and $15.27 \%$ at $2 \mathrm{~h}$, respectively. The mean fluorescence intensity (MFI) of the control group fluctuated slightly, but in general, the metabolic activity of $P$. fluorescens treated with linalool was significantly inhibited $(P<0.05)$.

\section{Effect of Linalool on Respiratory Chain Dehydrogenase}

Regarding the activity of respiratory chain dehydrogenase, the control group only slight growth compared to the initial data, whereas the $1 \mathrm{MIC}$ and 1/2 MIC groups had sharp declining trend, respectively (Figure 4D). Compared with the control group, the respiratory chain dehydrogenase activity of $1 / 2 \mathrm{MIC}$ and $1 \mathrm{MIC}$ groups were decreased by 35.58 and $76.37 \%$, respectively. The activity of respiratory chain dehydrogenase was significantly inversely correlated with concentration of linalool $(P<0.05)$.

\section{Effect of Linalool on Adenosine Triphosphatase (ATPase), Malic Acid Dehydrogenase (MDH), Succinic Acid Dehydrogenase (SDH), and Pyruvate Kinase (PK)} The effects of Linalool on the ATPase, MDH, SDH, and PK activities of $P$. fluorescens are shown in Figure 5. In general, the ATPase, SDH, $\mathrm{MDH}$, and $\mathrm{PK}$ activities of $P$. fluorescens showed some natural variation, whereas the values of the control group were significantly higher than those of the treatment group $(P<0.05)$. The ATPase activity of $P$. fluorescens treated with linalool decreased sharply within the first $2 \mathrm{~h}$, and the ATPase activity of the 1MIC group decreased to its minimum value of $1.0646 \mathrm{U} / \mathrm{mg}$ prot within $2 \mathrm{~h}$ (Figure 5A). The MDH activity of $P$. fluorescens in the two treatment groups increased in the first $0-1 \mathrm{~h}$ and reached maximum values of 3.4465 and $2.9314 \mathrm{U} / \mathrm{mg}$ prot, respectively and then decreased in the following1-2 $\mathrm{h}$ and reached minimum values of 0.5683 and $0.5162 \mathrm{U} / \mathrm{mg}$ prot, respectively (Figure 5B). Moreover, compared with the control group, the final $\mathrm{MDH}$ activity in the treatment group was significantly inhibited $(P<0.05)$. As shown in Figure 5C, the SDH activity of the control group varied across a narrow range, while the SDH activity in this group treated with linalool showed an increasing trend in $0-1 \mathrm{~h}$, and then decreased to 
A

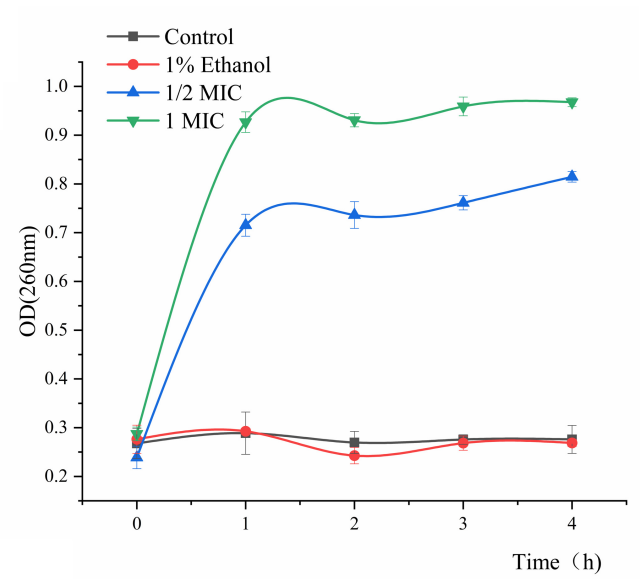

C

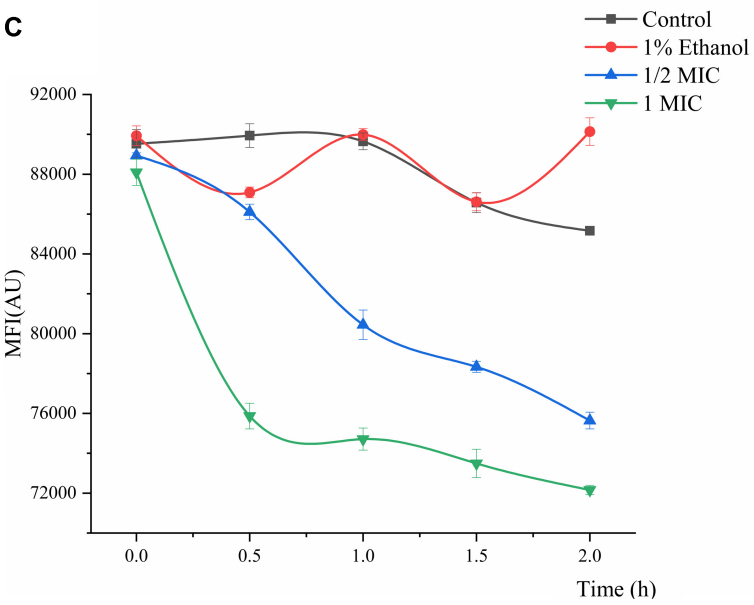

B

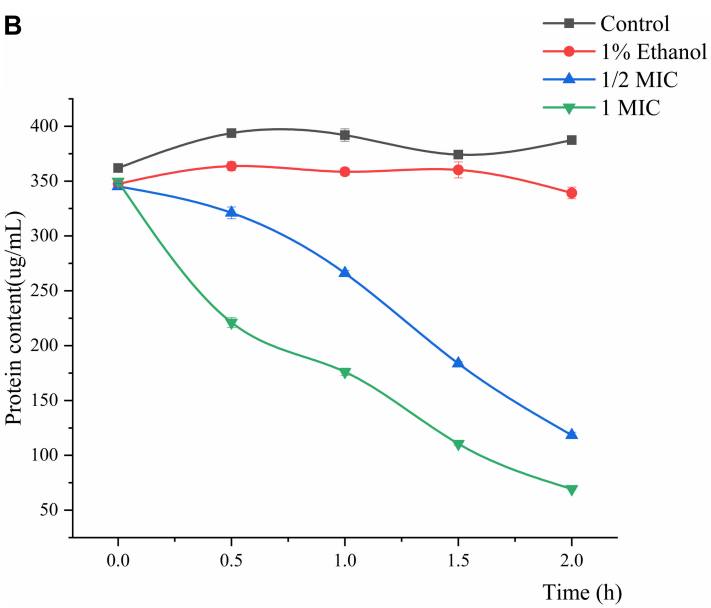

D

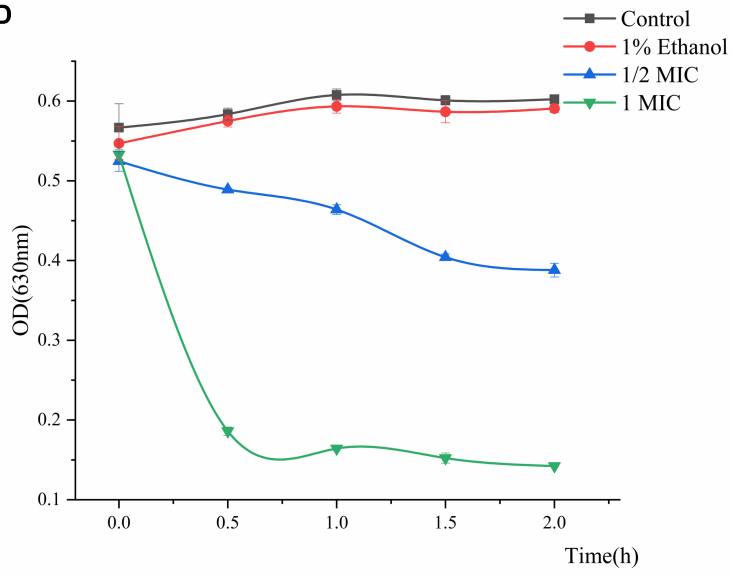

FIGURE 4 | Effect of treatment with different concentration of linalool on nucleic acid (A), intracellular protein (B), metabolic activity (C), and respiratory chain dehydrogenase (D) of $P$. fluorescens. Each value indicated the average of three independent determinations.

minimum value of 13.3136 and $7.3933 \mathrm{U} / \mathrm{mg}$ prot, respectively. The PK activity of the bacteria treated with linalool decreased significantly (Figure 5D) $(P<0.05)$. Compared with the control groups, after incubation with linalool for $2 \mathrm{~h}$ the PK activity of $P$. fluorescens was decreased by 34.12 and $52.57 \%$ in the $1 / 2$ MIC and 1 MIC.

\section{DISCUSSION}

EOs have been widely studied in the food preservative industry because they are natural and safe (Calo et al., 2015). Linalool, as the main component of many essential oils, has been proved to have potential antibacterial activity. Some studies have shown that the EOs of Cinnamomum camphora (contain 69.94\% linalool) (Wu et al., 2019), Gnaphlium affine (contain $10.62 \%$ linalool) (Zeng et al., 2011) and green huajiao (contain $28.2 \%$ linalool) (Diao et al., 2013) have a variety of antibacterial activities. The synergistic action of linalool and EOs can increase the antibacterial effect of EOs extracted from plants (Herman et al., 2016). Many authors have mentioned the antibacterial activity and antibacterial mechanism of essential oils, but the mechanism of action of plant EOs is very complicated due to its numerous components. In the present study, linalool, one of the main components of many plant EOs, was selected as an antibacterial agent to study its mechanism of action on P. fluorescens.

The results from the measurement of MIC and MBC, followed by the growth curve indicated that linalool had strong and consistent inhibiting effects on $P$. fluorescens (Figure 1). EOs (from Litsea cubeba leaf, Salvia mirzayanii, Zataria multiflora, Gnaphlium affine, Forsythia koreana) affect the structure of the cell envelope because it was found that the major antibacterial compositions of the EOs could penetrate through the cell wall and destroy the cytoplasmic membrane (Zeng et al., 2011; Kavoosi and Rabiei, 2015; Yang et al., 2015; Zomorodian et al., 2017; Nguyen et al., 2018). Scanning electron microscope (SEM) results showed that the cell morphology changed after linalool treatment (Figure 2). As a component of proton dynamics, MP is the potential difference between the inside and outside of biological cells, and it is closely related to the production of ATP. When the external environment of the cell changes, the ion concentration on opposite sides of the cell membrane will change, and the cell 

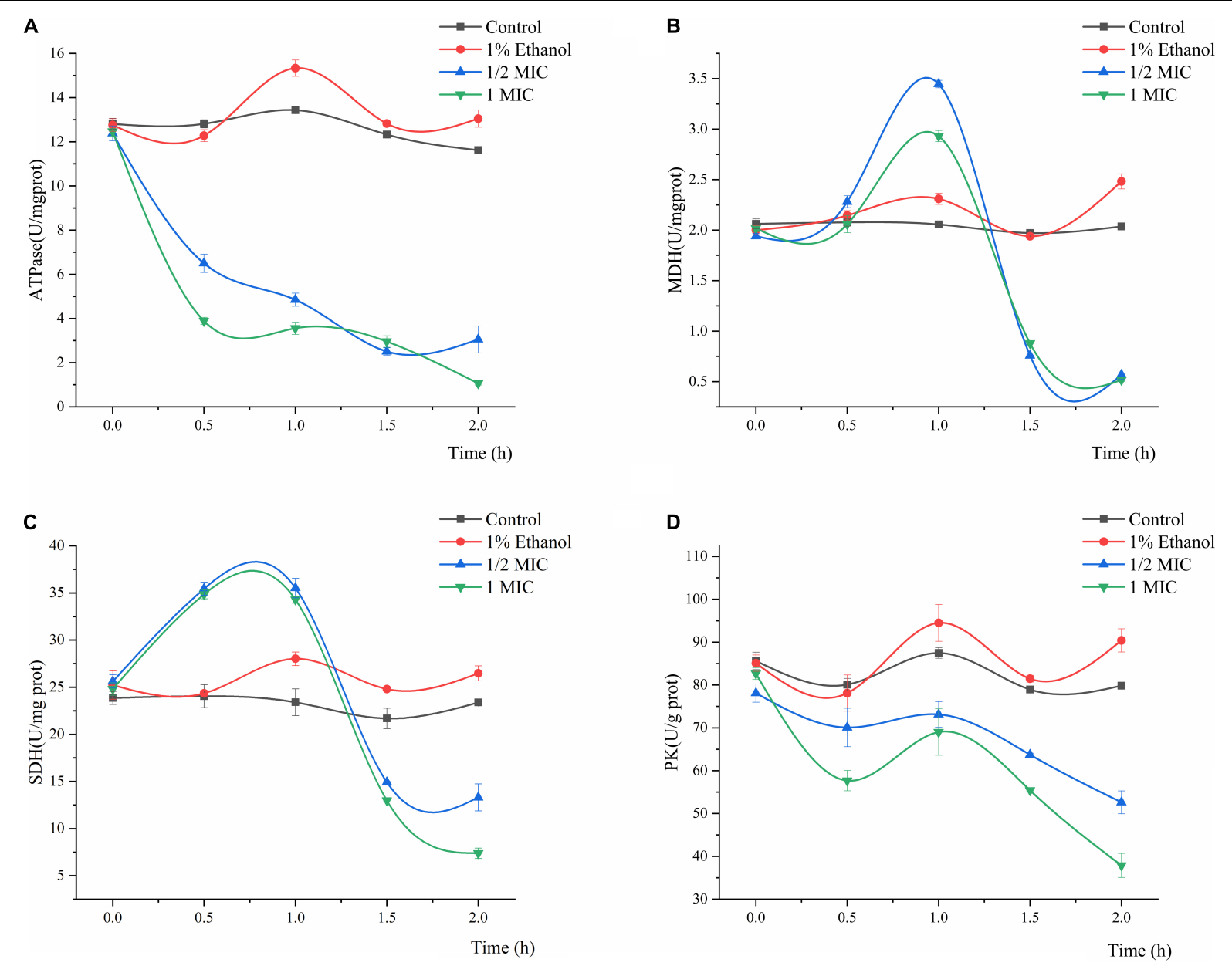

FIGURE 5 | Effect of treatment with different concentration of linalool on the activity of Adenosine triphosphatase (ATPase), Malic acid dehydrogenase (MDH), Succinic acid dehydrogenase (SDH), and Pyruvate kinase (PK). (A) ATPase of $P$. fluorescens, (B) MDH of $P$. fluorescens, (C) SDH of $P$. fluorescens, (D) PK of $P$. fluorescens. Each value indicated the average of three independent determinations.

membrane will depolarize, resulting in a change of membrane potential and thus affecting cell metabolism (Zhang et al., 2017; Fang et al., 2019). Therefore, the mechanism of antibacterial action was reflected by the determination of MP. In the present study, we measured the membrane potential of $P$. fluorescens exposed to linalool at 1/2MIC and MIC levels (Figure 3). The results clearly showed that the treatment with linalool could lead to cell depolarization and affect the production of ATP and reduce cellular metabolic activity.

Intracellular macromolecules were selected as another aspect to elucidate the mechanism of the antimicrobial action of linalool, because the integrity of the plasma membrane is a key factor in bacterial growth (Zhang et al., 2016). In normal cells, nucleic acids and proteins are macromolecules that present in the cell membrane and cytoplasm of bacteria. Nucleic acids carry unique genetic information and participate in translation, transcription, and DNA replication, while proteins provide major structural functions. Hu et al. (2019) found that treatment with Litsea cubeba EO ( $\beta$-Linalool 1.201\%) affected the integrity of the cell membrane of Staphylococcus aureus, which lead to the leakage of intracellular macromolecules which included nucleic acids and proteins. Our results showed that the intracellular leakage of macromolecules increased with the addition of linalool (Figures 4A,B). Therefore, linalool may act on the cell membrane and affect the integrity of the membrane, which caused nucleic acids and proteins to be released through the fault membrane, and resulted in increased intracellular leakage of macromolecules (Diao et al., 2014).

Metabolic respiration is considered to be the main energy producing process for the germination and growth of most microorganisms. Similarly, the respiratory system is one of the most common targets for antimicrobial agents (Yang et al., 2016). Resazurin is a type of weak fluorescent substance, which can be degraded by various oxidoreductases enzymes in cells into high fluorescence resorufin. Therefore, the metabolic activity of $P$. fluorescens can be characterized by the determination of the average fluorescence intensity after treatment with linalool (Zhu et al., 2019). The results showed that the average fluorescence intensity of $P$. fluorescens decreased after treatment with linalool, indicating that its metabolic capacity was weakened 
(Figure 4C). INT can be converted into water-insoluble and dark red iodonitrotetrazolium formazan (INF) by the bacterial respiratory chain dehydrogenase. INT has an absorption peak at $630 \mathrm{~nm}$, thus the loss of respiratory chain enzymatic activity can be assessed by the decreasing spectrophotometric value of INF (Sun et al., 2018). The results showed that the activity of respiratory chain dehydrogenase was inhibited after treatment with linalool, which was consistent with the results of the metabolic activity (Figure 4D). Bacteriostatic agents may break through the barrier of the extracellular membrane, peptidoglycan and periplasmic, and destroy respiratory chain dehydrogenase, thus inhibiting cellular respiration. The process has previously similar been reported for various antibacterial agents (Gomaa, 2017).
Adenosine triphosphate (ATP) is closely related to energy metabolism, cell function and life activities (Bajpai et al., 2013). ATPase is one of the most important enzymes to promote the production and metabolism of ATP. Its main mode of action is to provide cofactor and energy for cells, catalyze ATP to ADP, and thus provide energy for cells (Pei et al., 2019). The decline in ATPase activity may block the metabolism of carbohydrates, which can hamper cell growth and even lead to cell death (Lan et al., 2019). The decrease in the level of ATPase in P. fluorescens treated with linalool indicated that the activity of ATPase was destroyed by Linalool (Figure 5A). This phenomenon may lead to a decrease in the content of bacterial ATP, thus affecting the metabolic activity of the bacteria, which corresponds to the results of the MP analysis. Lin et al. (2018) also found that the

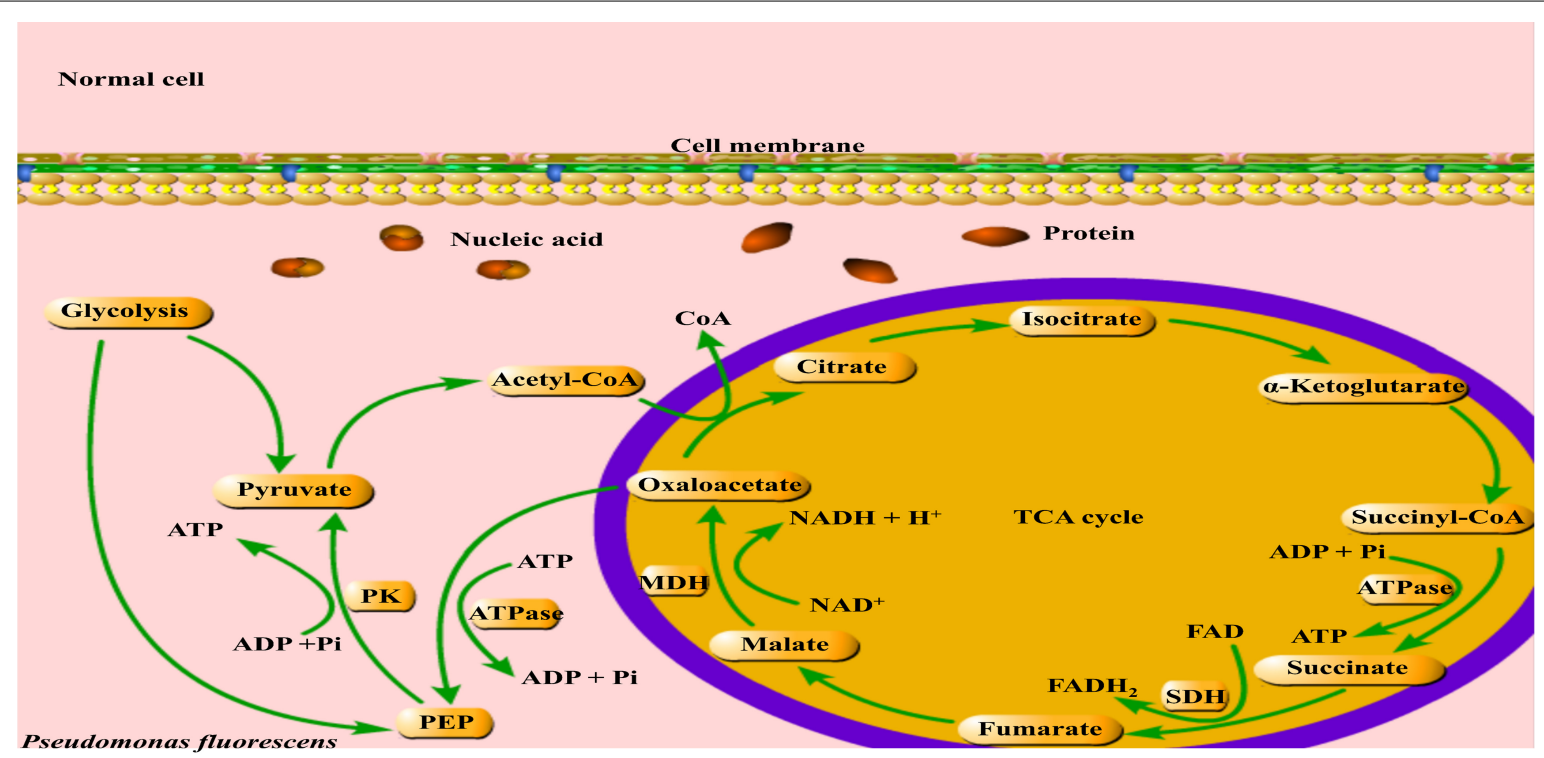

Linalool treatment

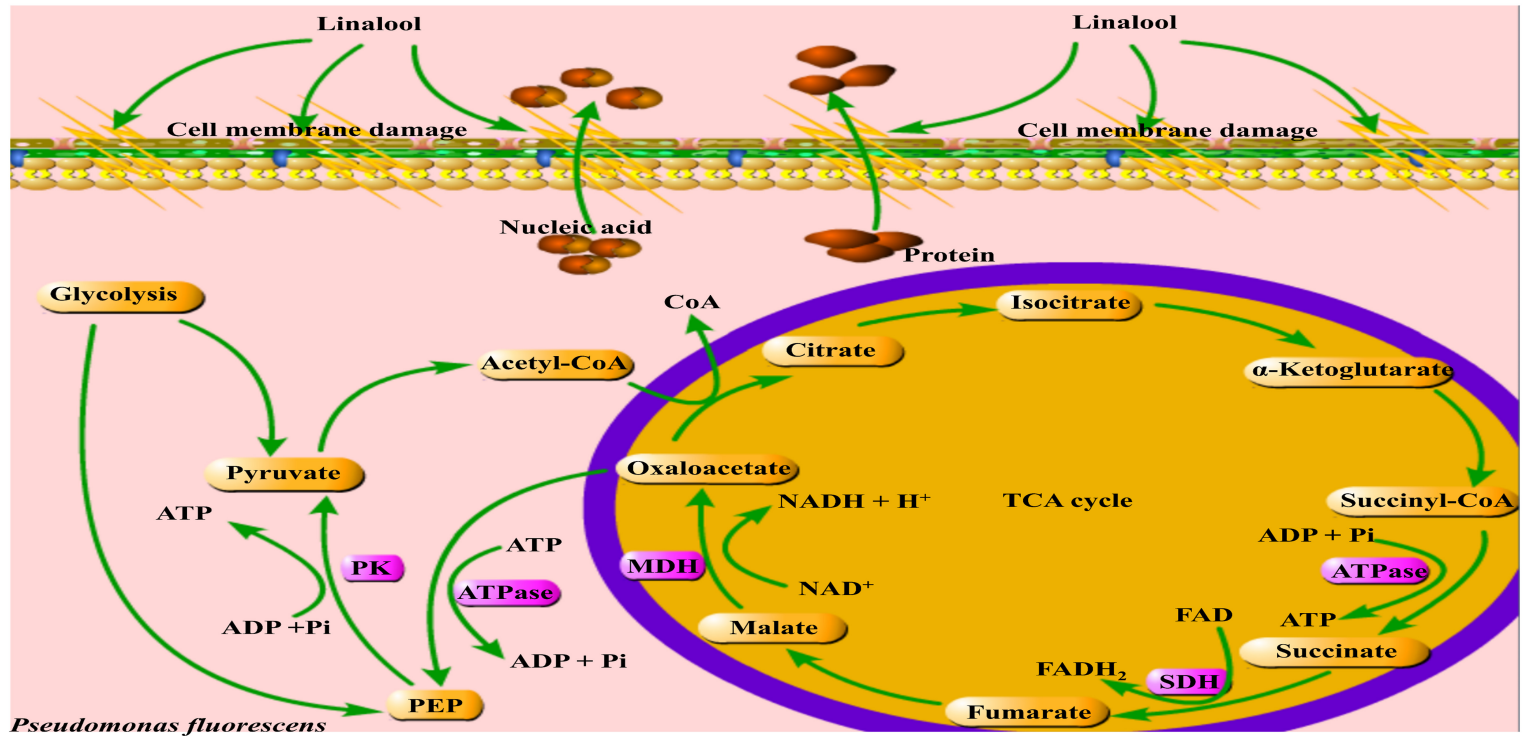

FIGURE 6 | Mechanisms of stress response in P. fluorescens. The purple frame represents the enzyme with significantly changed activity. 
bacteriostatic agent $\varepsilon$-PL could inhibit the respiration of Listeria monocytogenes by reducing the activity of ATPase.

In addition, the glycolysis pathway is closely related to the formation of ATP. Pyruvate kinase (PK) turns phosphoenolpyruvate and ADP, into ATP and pyruvate, respectively, and is one of the main rate-limiting enzymes in glycolysis (Wang et al., 2020). As shown in Figure 5, linalool treatment inhibited PK activity and prevented the generation of a pyruvate and ATP, thus affecting the metabolic activity of the bacteria. Under the action of pyruvate dehydrogenase system, the pyruvate undergoes oxidative decarboxylation to form acetylCoA. Acetyl-CoA reacts with oxaloacetic acid to form citric acid, which opens the tricarboxylic acid cycle (TCA). The TCA cycle, coupled with the energy in the mitochondria and interactions with a variety of anabolic pathways, is an essential metabolic pathway in all aerobic organisms (Fernie et al., 2004). As shown in Figure 6, $\mathrm{SDH}$ and $\mathrm{MDH}$ are both key enzymes involved in the TCA pathway in both eukaryotic and prokaryotic cells, and play a crucial role in energy metabolism in calls. SDH located on mitochondrial membrane, is the only multi-subunit enzyme involved in oxidative phosphorylation in the cell membrane. Succinate is formed from the conversion of succinyl-CoA, and then under the SDH catalytic succinate conversion to fumaric acid and simultaneously produces $\mathrm{FADH}_{2}$ (Akram, 2014). SDH links the respiratory metabolism and oxidative phosphorylation, therefore it is representative of the mitochondrial enzymes (Pei et al., 2019). Due to its important role in respiratory metabolism, $\mathrm{SDH}$ has become the target of some bacteriostatic agents. It was shown that SDH mutation was found in 14 pathogenic fungal resistant strains (Sierotzki and Scalliet, 2013). SDH activity of $P$. fluorescens after treatment with linalool indicated that bacterial respiration was weakened, which might lead to bacterial dysfunction (Figure 5C). MDH, another key enzyme in TCA, is widely found in mitochondria and bacterial cell membranes. It performs important metabolic functions in the aerobic energy production pathway, and is also involved in malic acid shuttling (Yao et al., 2011; Yang et al., 2016). MDH catalyzes L-malic acid to oxaloacetate and produces NADH (Pei et al., 2019). In the present study, treatment with linalool significantly reduced $\mathrm{MDH}$ activity (Figure 5B), suggesting that inhibition of the TCA cycle by linalool may result in an imbalance between aspartic acid malate shuttling and reduction equivalent.

Linalool has a strong and stable inhibitory effect on $P$. fluorescens. Treatment with linalool changed the morphology and structure of bacteria, destroyed the permeability of their cell membranes, induced the loss of intracellular macromolecules (include nucleic acids and proteins), inhibited the cells respiration rate, and reduced the activity of respiration related enzymes, including $\mathrm{MDH}, \mathrm{SDH}, \mathrm{PK}$, and ATPase. These

\section{REFERENCES}

Akram, M. (2014). Citric acid cycle and role of its intermediates in metabolism. Cell Biochem. Biophys. 68, 475-478. doi: 10.1007/s12013-013-9750-1

Aprotosoaie, A. C., Hăncianu, M., Costache, I.-I., and Miron, A. (2014). Linalool: a review on a key odorant molecule with valuable biological properties. Flav. Fragran. J. 29, 193-219. doi: 10.1002/ffj.3197 results suggest that linalool may inhibit metabolic respiration and disrupt enzyme activity related to energy production in $P$. fluorescens. As a result, cell membranes are destroyed, and cell metabolism is impaired.

\section{CONCLUSION}

With the attention of consumers on food safety, natural and safe EOs have become a popular subject of research in the field of food preservation. In the present study, the antibacterial activity and mechanism of linalool on $P$. fluorescens was explored. The results showed that linalool had a significant inhibitory effect on $P$. fluorescens. Linalool first acts on the cell membrane, causing damage to the structure and function of the cell membrane and causing the leakage of intracellular macromolecules. In addition, linalool seemed to have an effect on the activity of certain intracellular enzymes by inhibiting enzyme activity associated with the TCA cycle and glycolysis pathway. In addition, linalool also inhibited ATPase and respiratory chain dehydrogenase, thereby inhibiting ATP production and cellular respiration. In short, linalool acts in many of the above mentioned pathways, resulting in abnormal cellular structure and metabolic function, which results in cell death. Thus, linalool is a candidate as an effective natural food preservative and multifunctional food additive. We will continue this research and further explore the antibacterial mechanism of EOs to ensure the safe and effective application of natural antibacterial agents in the food industry.

\section{DATA AVAILABILITY STATEMENT}

The original contributions presented in the study are included in the article/supplementary material, further inquiries can be directed to the correponding author/s.

\section{AUTHOR CONTRIBUTIONS}

FG: writing-original draft. WXC and WJC: methodology. QC and QL: data curation. MZ: software. HC and YY: formal analysis. QZ: project administration. All authors contributed to the article and approved the submitted version.

\section{FUNDING}

This work was supported by the National Natural Science Foundation of China (grant no. 31760480).

Bajpai, V. K., Sharma, A., and Baek, K.-H. (2013). Antibacterial mode of action of Cudrania tricuspidata fruit essential oil, affecting membrane permeability and surface characteristics of food-borne pathogens. Food Control 32, 582-590. doi: 10.1016/j.foodcont.2013.01.032

Brenes, A., and Roura, E. (2010). Essential oils in poultry nutrition: main effects and modes of action. Anim. Feed Sci. Technol. 158, 1-14. doi: 10.1016/j.anifeedsci. 2010.03.007 
Caldera, L., and Franzetti, L. (2013). Effect of storage temperature on the microbial composition of ready-to-use vegetables. Curr. Microbiol. 68, 133-139. doi: 10. 1007/s00284-013-0430-6

Callaway, T. R., Carroll, J. A., Arthington, J. D., Edrington, T. S., Anderson, R. C., Ricke, S. C., et al. (2011). "Citrus products and their use against bacteria: potential health and cost benefits," in Nutrients Dietary Supplements and Nutriceuticals: Cost Analysis Versus Clinical Benefits, eds R. Watson, J. L. Gerald, and V. R. Preedy (New York, NY: Humana Press), 277-286.

Calo, J. R., Crandall, P. G., O’Bryan, C. A., and Ricke, S. C. (2015). Essential oils as antimicrobials in food systems - a review. Food Control 54, 111-119. doi: 10.1016/j.foodcont.2014.12.040

Cui, H., Zhang, C., Li, C., and Lin, L. (2018). Antimicrobial mechanism of clove oil on Listeria monocytogenes. Food Control 94, 140-146. doi: 10.1016/j.foodcont. 2018.07.007

Diao, M., Qi, D., Xu, M., Lu, Z., Lv, F., Bie, X., et al. (2018). Antibacterial activity and mechanism of monolauroyl-galactosylglycerol against Bacillus cereus. Food Control 85, 339-344. doi: 10.1016/j.foodcont.2017.10.019

Diao, W. R., Hu, Q. P., Feng, S. S., Li, W. Q., and Xu, J. G. (2013). Chemical composition and antibacterial activity of the essential oil from green huajiao (Zanthoxylum schinifolium) against selected foodborne pathogens. J. Agric. Food Chem. 61, 6044-6049. doi: 10.1021/jf4007856

Diao, W.-R., Hu, Q.-P., Zhang, H., and Xu, J.-G. (2014). Chemical composition, antibacterial activity and mechanism of action of essential oil from seeds of fennel (Foeniculum vulgare Mill.). Food Control 35, 109-116. doi: 10.1016/j. foodcont.2013.06.056

Faleiro, M. L. (2011). "The mode of antibacterial action of essential oils," in Science Against Microbial Pathogens: Communicating Current Research and Technological Advances, ed. A. Méndez-Vilas (Portugal: University of Algarve), 1143-1156.

Fang, Z., Xu, L., Lin, Y., Cai, X., and Wang, S. (2019). The preservative potential of octopus scraps peptides-zinc chelate against Staphylococcus aureus: its fabrication, antibacterial activity and action mode. Food Control 98, 24-33. doi: 10.1016/j.foodcont.2018.11.015

Fernie, A. R., Carrari, F., and Sweetlove, L. J. (2004). Respiratory metabolism: glycolysis, the TCA cycle and mitochondrial electron transport. Curr. Opin. Plant Biol. 7, 254-261. doi: 10.1016/j.pbi.2004.03.007

Fratianni, F., De Martino, L., Melone, A., De Feo, V., Coppola, R., and Nazzaro, F. (2010). Preservation of chicken breast meat treated with thyme and balm essential oils. J. Food Sci. 75, M528-M535. doi: 10.1111/j.1750-3841.2010.01 791.x

Gomaa, E. Z. (2017). Silver nanoparticles as an antimicrobial agent: a case study on Staphylococcus aureus and Escherichia coli as models for Gram-positive and gram-negative bacteria. J. Gen. Appl. Microbiol. 63, 36-43. doi: 10.2323/jgam. 2016.07.004

Herman, A., Tambor, K., and Herman, A. (2016). Linalool affects the antimicrobial efficacy of essential oils. Curr. Microbiol. 72, 165-172. doi: 10.1007/s00284-0150933-4

Hu, W., Li, C., Dai, J., Cui, H., and Lin, L. (2019). Antibacterial activity and mechanism of Litsea cubeba essential oil against methicillin-resistant Staphylococcus aureus (MRSA). Indust. Crops Prod. 130, 34-41. doi: 10.1016/ j.indcrop.2018.12.078

Jin, L., Liu, X., Bian, C., Sheng, J., Song, Y., and Zhu, Y. (2020). Fabrication linaloolfunctionalized hollow mesoporous silica spheres nanoparticles for efficiently enhance bactericidal activity. Chin. Chem. Lett. 31, 2137-2141. doi: 10.1016/j. cclet.2019.12.020

Kang, J. W., Kim, S. S., and Kang, D. H. (2018). Inactivation dynamics of $222 \mathrm{~nm}$ krypton-chlorine excilamp irradiation on Gram-positive and Gram-negative foodborne pathogenic bacteria. Food Res. Int. 109, 325-333. doi: 10.1016/j. foodres.2018.04.018

Kavoosi, G., and Rabiei, F. (2015). Zataria multiflora: chemical and biological diversity in the essential oil. J. Essent. Oil Res. 27, 428-436. doi: 10.1080/ 10412905.2015.1031917

Kumar, H., Franzetti, L., Kaushal, A., and Kumar, D. (2019). Pseudomonas fluorescens: a potential food spoiler and challenges and advances in its detection. Ann. Microbiol. 69, 873-883. doi: 10.1007/s13213-019-01501-7

Lan, W., Zhang, N., Liu, S., Chen, M., and Xie, J. (2019). Epsilon-Polylysine inhibits Shewanella putrefaciens with membrane disruption and cell damage. Molecules 24:727. doi: $10.3390 /$ molecules 24203727
Lin, L., Gu, Y., Li, C., Vittayapadung, S., and Cui, H. (2018). Antibacterial mechanism of $\varepsilon$-Poly-lysine against Listeria monocytogenes and its application on cheese. Food Control 91, 76-84. doi: 10.1016/j.foodcont.2018. 03.025

Liu, X., Cai, J., Chen, H., Zhong, Q., Hou, Y., Chen, W., et al. (2020). Antibacterial activity and mechanism of linalool against Pseudomonas aeruginosa. Microb. Pathog. 141:103980. doi: 10.1016/j.micpath.2020.103980

Nguyen, H. V., Meile, J. C., Lebrun, M., Caruso, D., Chu-Ky, S., and Sarter, S. (2018). Litsea cubeba leaf essential oil from Vietnam: chemical diversity and its impacts on antibacterial activity. Lett. Appl. Microbiol. 66, 207-214. doi: 10.1111/lam.12837

Pei, Q., Li, Y., Ge, X., and Tian, P. (2019). Multipath effects of berberine on peach Brown rot fungus Monilinia fructicola. Crop Protect. 116, 92-100. doi: 10.1016/j.cropro.2018.10.017

Pereira, I., Severino, P., Santos, A. C., Silva, A. M., and Souto, E. B. (2018). Linalool bioactive properties and potential applicability in drug delivery systems. Coll. Surf. B Biointerf. 171, 566-578. doi: 10.1016/j.colsurfb.2018.0 8.001

Prakash, B., Kedia, A., Mishra, P. K., and Dubey, N. K. (2015). Plant essential oils as food preservatives to control moulds, mycotoxin contamination and oxidative deterioration of agri-food commodities - Potentials and challenges. Food Control 47, 381-391. doi: 10.1016/j.foodcont.2014.0 7.023

Quintieri, L., Fanelli, F., and Caputo, L. (2019). Antibiotic resistant Pseudomonas Spp. spoilers in fresh dairy products: an underestimated risk and the control strategies. Foods 8:372. doi: 10.3390/foods8090372

Reichler, S. J., Trmcic, A., Martin, N. H., Boor, K. J., and Wiedmann, M. (2018). Pseudomonas fluorescens group bacterial strains are responsible for repeat and sporadic postpasteurization contamination and reduced fluid milk shelf life. J. Dairy Sci. 101, 7780-7800. doi: 10.3168/jds.2018-14438

Shu, H., Chen, H., Wang, X., Hu, Y., Yun, Y., Zhong, Q., et al. (2019). Antimicrobial activity and proposed action mechanism of 3-Carene against Brochothrix thermosphacta and Pseudomonas fluorescens. Molecules 24:3246. doi: 10.3390/ molecules 24183246

Sierotzki, H., and Scalliet, G. (2013). A review of current knowledge of resistance aspects for the next-generation succinate dehydrogenase inhibitor fungicides. Phytopathology 103, 880-887.

Sun, X.-H., Zhou, T.-T., Wei, C.-H., Lan, W.-Q., Zhao, Y., Pan, Y.-J., et al (2018). Antibacterial effect and mechanism of anthocyanin rich Chinese wild blueberry extract on various foodborne pathogens. Food Control 94, 155-161. doi: 10.1016/j.foodcont.2018.07.012

Wang, Y., Liu, R., Hou, Q., Tian, X., Fan, X., Zhang, W., et al. (2020). Comparison of activity, expression and S-nitrosylation of glycolytic enzymes between pale, soft and exudative and red, firm and non-exudative pork during post-mortem aging. Food Chem. 314:126203. doi: 10.1016/j.foodchem.2020.126203

Wu, K., Lin, Y., Chai, X., Duan, X., Zhao, X., and Chun, C. (2019). Mechanisms of vapor-phase antibacterial action of essential oil from Cinnamomum camphora var. linaloofera Fujita against Escherichia coli. Food Sci. Nutr. 7, 2546-2555. doi: $10.1002 / \mathrm{fsn} 3.1104$

Yang, S., Liu, L., Li, D., Xia, H., Su, X., Peng, L., et al. (2016). Use of active extracts of poplar buds against Penicillium italicum and possible modes of action. Food Chem. 196, 610-618. doi: 10.1016/j.foodchem.2015.09.101

Yang, X. N., Khan, I., and Kang, S. C. (2015). Chemical composition, mechanism of antibacterial action and antioxidant activity of leaf essential oil of Forsythia koreana deciduous shrub. Asian Pac. J. Trop. Med. 8, 694-700. doi: 10.1016/j. apjtm.2015.07.031

Yao, Y.-X., Li, M., Zhai, H., You, C.-X., and Hao, Y.-J. (2011). Isolation and characterization of an apple cytosolic malate dehydrogenase gene reveal its function in malate synthesis. J. Plant Physiol. 168, 474-480. doi: 10.1016/j.jplph. 2010.08.008

Zeng, W. C., Zhu, R. X., Jia, L. R., Gao, H., Zheng, Y., and Sun, Q. (2011). Chemical composition, antimicrobial and antioxidant activities of essential oil from Gnaphlium affine. Food Chem. Toxicol. 49, 1322-1328. doi: 10.1016/j.fct. 2011.03.014

Zhang, L.-L., Zhang, L.-F., Hu, Q.-P., Hao, D.-L., and Xu, J.-G. (2017). Chemical composition, antibacterial activity of Cyperus rotundus rhizomes essential oil against Staphylococcus aureus via membrane disruption and apoptosis pathway. Food Control 80, 290-296. doi: 10.1016/j.foodcont.2017.05.016 
Zhang, Y., Liu, X., Wang, Y., Jiang, P., and Quek, S. (2016). Antibacterial activity and mechanism of cinnamon essential oil against Escherichia coli and Staphylococcus aureus. Food Control 59, 282-289. doi: 10.1016/j.foodcont.2015. 05.032

Zhu, Y., Li, C., Cui, H., and Lin, L. (2019). Antimicrobial mechanism of pulsed light for the control of Escherichia coli $\mathrm{O} 157: \mathrm{H} 7$ and its application in carrot juice. Food Control 106:106751. doi: 10.1016/j.foodcont.2019.106751

Zomorodian, K., Moein, M., Pakshir, K., Karami, F., and Sabahi, Z. (2017). Chemical composition and antimicrobial activities of the essential oil from Salvia mirzayanii leaves. J. Evid. Based Complement. Altern. Med. 22, 770-776. doi: $10.1177 / 2156587217717414$
Conflict of Interest: The authors declare that the research was conducted in the absence of any commercial or financial relationships that could be construed as a potential conflict of interest.

Copyright (C) 2021 Guo, Chen, Liang, Zhang, Chen, Chen, Yun, Zhong and Chen. This is an open-access article distributed under the terms of the Creative Commons Attribution License (CC BY). The use, distribution or reproduction in other forums is permitted, provided the original author(s) and the copyright owner(s) are credited and that the original publication in this journal is cited, in accordance with accepted academic practice. No use, distribution or reproduction is permitted which does not comply with these terms. 